\title{
Factors Related to Seizure in Tramadol Poisoning and Its Blood Concentration
}

\author{
Fakhreddin Taghaddosinejad • Omid Mehrpour • \\ Reza Afshari • Alireza Seghatoleslami • \\ Mohammad Abdollahi • Richard C. Dart
}

Published online: 7 July 2011

(C) American College of Medical Toxicology 2011

\begin{abstract}
This study examines the relation between seizure and plasma tramadol concentration in patients with tramadol poisoning, as a novel centrally acting analgesic used for the treatment of mild to severe pain. All patients admitted with a history of tramadol overdose accompanied by unconsciousness or seizures referred to Baharloo Hospital Poison Center, Tehran, Iran from March 2008 to March 2009 were included. Demographic information, clinical findings, and blood tramadol concentrations were studied. There were 401 patients with a history of tramadol overdose; 121 (30.2\%) with a history of seizure and $14(3.5 \%)$ with a history of unconsciousness were included. Most of overdoses involved men (83\%). The mean age was 22.9 years (range, 14-50 years). Intentional overdose was the most common mode of poisoning (51.9\%). The mean dose ingested was $1,511 \mathrm{mg}$ (SD, 1,353; range, 200-7,000). Mean back-extrapolated
\end{abstract}

F. Taghaddosinejad $\cdot$ A. Seghatoleslami

Department of Forensic Medicine and Toxicology, Faculty of

Medicine, Tehran University of Medical Sciences,

Tehran, Iran

O. Mehrpour

Department of Clinical Toxicology and Forensic Medicine, Vali-Asr Hospital, Birjand University of Medical Sciences, Birjand, Iran

O. Mehrpour

Medical Toxicology and Drug Abuse Research Group, Birjand

University of Medical Sciences,

Birjand, Iran

R. Afshari

Medical Toxicology Research Centre,

Mashhad University of Medical Sciences,

Mashhad, Iran tramadol blood concentrations were $3,843 \mathrm{ng} / \mathrm{mL}(3,715$; 269-20,049). Back-extrapolated blood concentrations were correlated with dose $(r=0.313 ; P<0.001)$ as well as blood concentration levels $(r=0.801 ; P<0.001)$. Seizure was significantly correlated to higher reported dose $(P<0.001)$ and tramadol only to overdose $(P<0.001)$. However, it was neither related to higher tramadol blood concentrations, nor related to time elapsed, age, sex, history of addiction, and observed Glasgow Coma Scale of patients. Most patients experienced just one seizure $(76 \%)$. The tramadol-induced seizure is dose dependent. Although higher doses of tramadol was related to higher blood concentration, blood tramadol concentrations was not associated with seizure.

Keywords Tramadol $\cdot$ Seizure $\cdot$ Blood concentration . Poisoning

\footnotetext{
M. Abdollahi

Faculty of Pharmacy, and Pharmaceutical Sciences Research Center, Tehran University of Medical Sciences (TUMS), Tehran 1417614411, Iran

R. C. Dart

Rocky Mountain Poison and Drug Center,

Denver Health and Hospital Authority

and University of Colorado School of Medicine,

Denver, USA

O. Mehrpour $(\bowtie)$

Department of Clinical Toxicology and Forensic Medicine,

Faculty of Medicine, Birjand University of Medical Sciences,

Ghaffari Avenue,

Birjand, Iran

e-mail: omehrpour@razi.tums.ac.ir

O. Mehrpour

e-mail: omid.mehrpour@yahoo.com.au
} 


\section{Introduction}

Tramadol is a novel centrally acting analgesic used for the treatment of mild to severe pain $[1,2]$. It has been approved in some countries since 1980 and become the most prescribed opioid worldwide [3, 4]. Tramadol was authorized by the Iran Drug Selecting Committee in 2002 as an analgesic. During this period, it has been abused especially by the younger population [5].

Tramadol binds weakly to the $\mu$-opioid receptor and also inhibits reuptake of monoamines such as serotonin and norepinephrine [2-5]. Most of the analgesic effect of tramadol may be secondary to non-opioid properties, via the central monoaminergic pathways. The most common route of administration of tramadol is oral, and its volume of distribution is about $3 \mathrm{~L} / \mathrm{kg}$.

Opioid dependency, overdose, and related complications are common in Iran in various age groups and gender [6, 7]. In recent years, tramadol overdose has become one of the most common causes of poisoning admissions to emergency departments in this country [5, 8-10]. An increased rate of seizure due to tramadol poisoning has also been observed. It is reported that $15 \%$ to $35 \%$ of patients with tramadol overdose experience seizure $[5,11]$. The exact mechanism of tramadol in induction of seizure remains to be elucidated yet. Research indicates that in high concentrations tramadol exerts an inhibitory effect on gammaaminobutyric acid (GABA) receptors $[12,13]$. Inhibition of GABA receptors has been found to potentiate the severity of seizures in animal models $[13,14]$. In addition, GABA receptor inhibition induced by tramadol can be secondary to its opioid receptor agonist activity [13], and continuing this agonist activity on opioid receptor has been proven to precipitate seizure due to inhibition of GABA pathways $[15,16]$. In addition to overdose, seizures have been reported in patients receiving tramadol at recommended doses [17]. Tramadol may also increase the seizure risk in patients receiving other medications such as tricyclic antidepressants, phenothiazines, and selective serotonin reuptake inhibitors [11]. While tramadol-related seizures can be controlled by diazepam, they are not responsive to naloxone, and tramadol-induced seizures can be precipitated by administration of naloxone, at high tramadol doses [18].

The analgesic effect of tramadol is dose dependent. The relation between serum concentrations and analgesia varies between individuals [1, 5]. It is estimated that serum concentrations of 100 to $300 \mathrm{ng} / \mathrm{mL}$ are needed for analgesia [3-5, 19].

In medical literature, no study in regard to blood concentrations of tramadol-induced seizures is available. The aim of this study is to find relation between seizure and plasma tramadol concentration in patients with tramadol overdose. Since Baharloo hospital in Tehran is one of the referral hospitals for treatment of poisoned patients, data obtained from this center can be extrapolated to other parts of the country [20].

\section{Methods}

Tehran University of Medical Sciences review board approved the ethical aspects of the study in a prospective case series with tramadol overdose. The study was done in Baharloo Hospital Poison Center, Tehran, Iran from, 21st March 2008 to 21st March 2009.

This was a prospective observational study. All patients who admitted to the hospital with a history of tramadol overdose confirmed to ingest more than the recommended therapeutic dose and experiencing a seizure or loss of consciousness were included in the study.

Data were collected in a predesigned questionnaire, which was completed by one of the clinical toxicology residents who was blind to the outcome of the analysis. Information was obtained from conscious patients if possible and from their relatives. Validity of data collection was controlled by one of the consultants for the first ten cases. During study period, a total of 401 patients with a history of tramadol overdose were admitted and treated in the emergency department. Of these, 266 were mild cases (fully alert without any signs or symptoms of poisoning before admission and after adequate remaining in the ED) who were managed as outpatient and excluded from final analysis. Declaring tramadol and co-ingested compounds as the cause of overdose were considered enough to include the subjects in the study. Demographic information, cause of poisoning, ingested dose, time elapsed from ingestion, seizure, history of tramadol abuse, history of illicit abuse or dependency, previous history of suicide, frequency of seizures, family history of seizure, previous history of tramadol poisoning, and co-ingestion were collected at the time of admission. Glasgow Coma Scale (GCS) on admission was also documented by the residents.

Patients received supportive, symptomatic, and conservative treatments under the direction of the treating physician, including gastrointestinal decontamination, activated charcoal, diazepam, chlordiazepoxide, naloxone, and fluids.

Blood samples for tramadol measurements were collected on admission and the elapsed time between ingestion and blood sampling was recorded. Gas chromatography (YOUNGLIN, 2000, KOREA) was used to measure plasma tramadol concentration. Standard curves from 100 to $10,000 \mathrm{ng} / \mathrm{mL}$ concentration were used to estimate plasma tramadol levels.

Blood levels at the time of tramadol use were calculated via back extrapolation to the time of ingestion using 
information from healthy volunteers of the same population [21]. All calculation was performed manually with the help of Microsoft ${ }^{\circledR}$ Office Excel 2003. Further analysis has been performed using these estimated blood levels.

Prevalence of seizure was calculated based on all cases. All cases with seizure were admitted. The rest of the measurements were presented from admitted cases.

Appropriate parametric and non parametric tests were used. Two tailed tests with a $P$ value less than 0.05 were considered to be statistically significant.

\section{Results}

Demographic

One hundred thirty-five cases were included in this study, of them, $121(30.2 \%)$ patients had a history of a witnessed seizure and $14(3.5 \%)$ patients had a history of loss of consciousness.

Most of the patients were men $(83 \%)$ with a mean age of 22.9 years (range, 14 to 50 years). Young adults (2130 years) comprised $50 \%$ of cases and $43 \%$ of cases were 11-20 years old. There were no significant difference between males and females in terms of age, reported tramadol dose, time between ingestion, seizure, and GCS.

Intentional overdose was the most common type of poisoning $(51.9 \%)$, followed by recreational abuse $(29.6 \%)$, replacement for opioid agents $(7.4 \%)$, other therapeutic purposes (7.4\%) and accidental poisoning (3.7\%) (Table 1). The route of poisoning in all patients was oral.

\section{Past Medical History}

Among 135 cases, 30 patients $(22.2 \%)$ had a history of previous admission due to tramadol poisoning. Sixteen of these 30 patients $(53.3 \%)$ reported a previous history of seizure due to tramadol poisoning. History of previous

Table 1 Distribution of admitted cases according to the etiology of tramadol abuse

\begin{tabular}{lc}
\hline Etiology of tramadol abuse & Frequency (\%) \\
\hline Intentional & $70(51.9)$ \\
For euphoria & $40(29.6)$ \\
Replacement of opioid & $10(7.4)$ \\
Therapeutic & $9(6.9)$ \\
Accidental & $5(3.7)$ \\
Other & $1(0.7)$ \\
Total & $135(100)$ \\
\hline
\end{tabular}

seizure and history of admission due to tramadol poisoning were significantly correlated $(P=0.01)$. Family history of seizure was reported in $2 \%$ of cases.

A history of chronic tramadol abuse was found in $25 \%$ of cases. Opioid dependency was reported in $20 \%$ of cases. The most commonly used illicit agents were opium (31\%), crack-heroin $(23 \%)$, crystal heroin $(3 \%)$, heroin $(2.2 \%)$, cannabis $(2.2 \%)$, and others $(1.5 \%)$. Thirty patients $(22.2 \%)$ reported history of previous suicide attempt.

A benzodiazepine drug was co-ingested in ten patients. One of these had a positive family history of seizure; one had a previous history of seizure; and two cases had a history of previous poisoning with tramadol and admission to hospital. Among these ten cases, four (40\%) had seizure, one episode only in each case.

\section{Blood Tramadol Concentrations}

The mean (SD; Min-Max) reported dose was $1,511 \mathrm{mg}$ (1,353 mg; 200-7,000 mg). Time elapsed between ingestion and blood sampling was $5.2(3.1 ; 1-16.5) \mathrm{h}$. Mean back-extrapolated tramadol blood concentrations were 3,843 (3,715 ng/mL, 269-20,049 ng/mL). In all patients, extrapolated blood tramadol concentrations were significantly related to reported dose $(r=0.318, P<0.001)$. The reported tramadol dose in relation to extrapolated blood tramadol levels is shown in Fig. 1. Extrapolated blood tramadol levels in patients with or without seizure are shown in Fig. 2. The results are clustered based on the gender of patients. Mean tramadol blood concentrations in males were higher, 4,029 $(3,945) \mathrm{ng} / \mathrm{mL}$, but not significantly different from females 2,969 $(2,211) \mathrm{ng} / \mathrm{mL}$. Backextrapolated blood concentrations was correlated with reported dose $(r=0.313, P<0.001)$ as well as blood

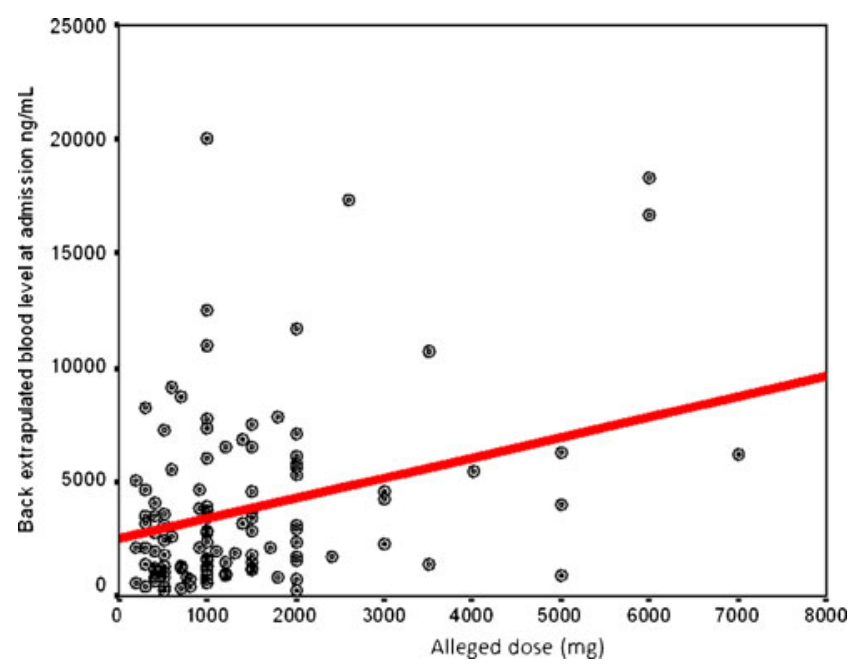

Fig. 1 Reported tramadol dose in relation to blood tramadol concentration $(n=131)$ 

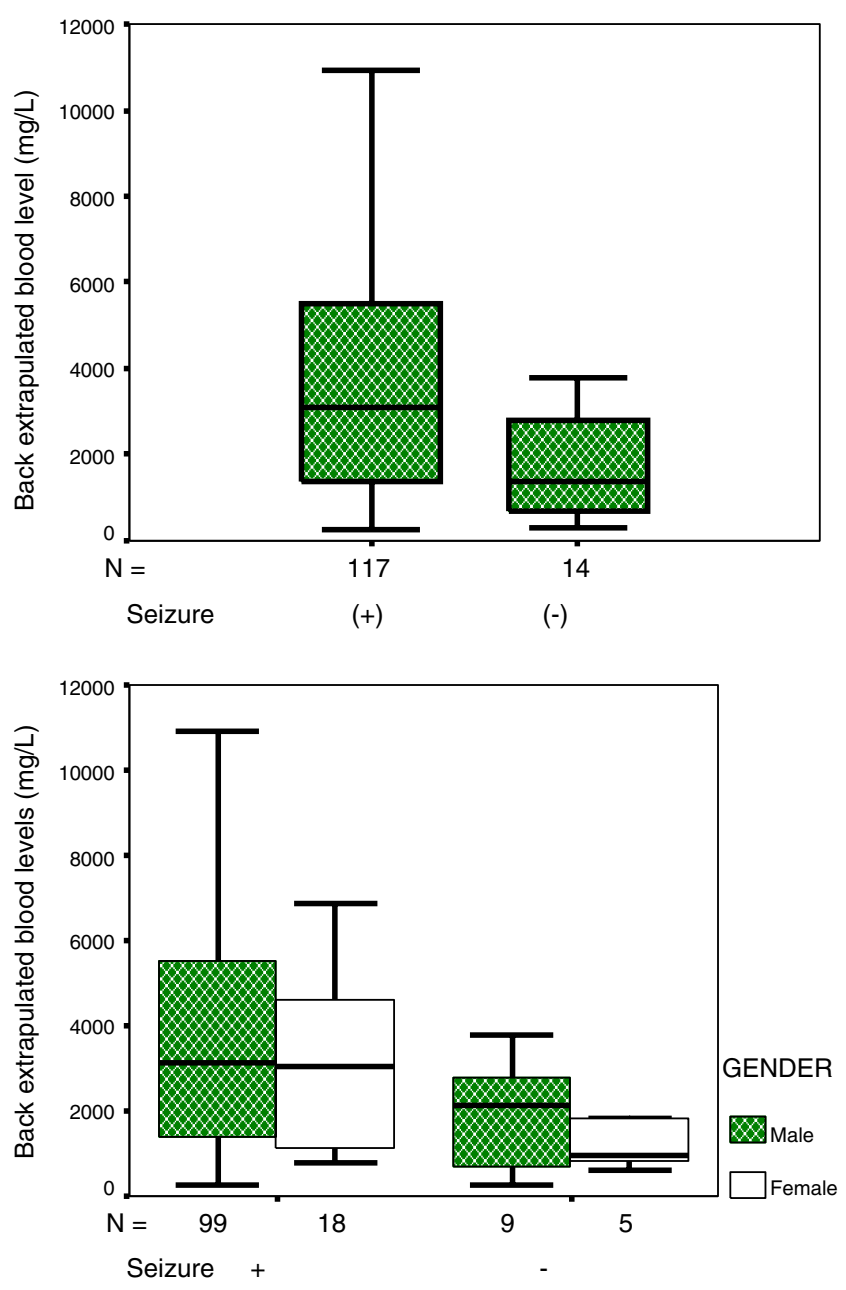

Fig. 2 Blood tramadol concentration in patients with or without seizure. The results are clustered on the basis of gender of patients (extreme cases are omitted from the figure; $n=131$ )

concentration $(r=0.801, P<0.001)$. Age, reported dose, time elapsed between ingestion, and blood samplings were not significantly different between males and females. Thirteen percent of these cases co-ingested other medications or chemicals, from which benzodiazepines were the most common (Table 2).

Table 2 Co-ingested drugs among admitted cases $(n=17)$

\begin{tabular}{lc}
\hline Drug & Frequency (\%) \\
\hline Benzodiazepine & $10(58.8)$ \\
NSAIDs & $3(17.6)$ \\
Methyl amphetamine & $2(11.8)$ \\
Tricyclic antidepressant (TCA) & $1(5.9)$ \\
Acetaminophen & $1(5.9)$ \\
Total & $17(100.0)$ \\
\hline
\end{tabular}

NSAIDs nonsteroidal anti-inflammatory drugs

\section{Clinical Findings}

Mean GCS on admission was $14(1.2 ; 7-15)$; most of which referred while fully alert with GCS of 15 (56.3\%).

Prevalence of seizure in the entire group of patients with tramadol overdose was $30 \%$ which happened on average $2.6(2.0 ; 0.3-12)$ hours after exposure; however $95 \%$ of seizures occurred in the first six hours after ingestion. Seizure was significantly correlated to higher reported dose $(P<0.001)$ and tramadol-alone overdose $(P<0.001)$. Seizure was not correlated to higher blood tramadol concentrations $(P=0.104)$, nor related to time elapsed, age, sex, history of addiction, and observed GCS of patients.

Majority of patients experienced one episode of seizure (76\%), followed by two episodes in $11.9 \%$. In $2 \%$ of cases, three or more episodes were observed.

\section{Discussion}

We observed that intentional overdose was the most common mode of tramadol poisoning in our study population. Young males were involved more frequently than females, which is in agreement with other studies in Iran and other countries $[5,9,22]$. The results show that ingestion of tramadol was the only route of intoxication. This is probably because tablets are widely available in Iranian private pharmacies [23]. Dispersion of injectable forms of tramadol is limited to non-private pharmacies and hospitals.

Among cases, $45 \%$ were long-term abusers of tramadol, indicating the potential for abuse. This finding is in contrast with some other reports in which tramadol has been reported to have low risk for abuse [24]. In Iran, however, tramadol has been reported to be increasingly abused by opioid dependent subjects [8]. It seems that the higher prevalence of tramadol abuse in this country may be because of less availability of other legal opioids such as hydrocodone or oxycodone.

In this study, $20 \%$ of cases were opioid dependent or abusers of other illicit agents at the same time, particularly opium. This is probably due to high availability of illicit opioids in Iran [6].

Seizure occurred in almost one third of tramadol overdoses (135 out of 401), which is inconsistent with previous reports [5, 9, 25]; although lower prevalence of seizure has been reported $[8,26]$. In this study, the smallest reported dose associated with seizure was $200 \mathrm{mg}$ (was seen in four patients). This is lower than previously published studies, in which the smallest dose of tramadol-associated seizure was reported to be $300 \mathrm{mg}[5,27]$.

Seizures caused by tramadol are typically of tonic-clonic type $[5,9,25]$, despite the fact that tramadol at clinically 
relevant doses may slightly suppress the severity of seizures [27]. Much of the toxicity in tramadol overdose can be attributed to the monoamine uptake inhibition rather than its opioid effects [8].

In the present study, time of seizure varied from $20 \mathrm{~min}$ to $12 \mathrm{~h}$ post-ingestion; however, $95 \%$ of seizures occurred in the first $6 \mathrm{~h}$ after ingestion, which is in agreement with studies conducted by Mehrpour et al. [28], Marquardt et al. [27], Jovanović-Cupić et al. [24], and Talaie et al. [9].

Single seizure was the most frequent one that happened in $84.3 \%$ of cases while multiple seizures were seen in $15.7 \%$ of cases, which is similar to other studies [24].

Previous history of seizure was reported in $15 \%$ of the patients. Although this implies that it is a risk factor for seizure, previous seizure in 16 patients of these 21 patients was because of previous tramadol poisoning. In our study, just $1.5 \%$ of cases had family history of seizure. Although it seems a weak risk factor for occurrence of seizure in these patients, prescribing tramadol for patients with previous history of tramadol poisoning should be performed cautiously.

We observed co-ingestion in 13\% of cases. Other studies have shown that co-ingestion with tramadol increases the risk of poisoning including seizure in cases of tricyclic antidepressants and SSRIs co-ingestions [11]. There is evidence that mortality due to tramadol poisoning is higher when co-ingested with benzodiazepine [1, 5]. In our study, the most co-ingested medications were benzodiazepines. No deaths, however, observed in this case series.

In our study, back-extrapolated tramadol blood concentrations varied from 269 to $20,049 \mathrm{ng} / \mathrm{mL}$ with a mean concentration of $3,843(3,715) \mathrm{ng} / \mathrm{mL}$. Majority of similar reports evaluated postmortem blood tramadol levels [29, 30].

Seizures were more frequent in higher reported doses suggesting seizure as a dose-dependent characteristic of tramadol overdose. Reported ingested dose and blood tramadol concentration were also correlated, but there was poor correlation between seizure and blood tramadol concentration. As a result, reported dose of tramadol overdose seems to be a good indicator to predict seizure in our population. This is not consistent with a previous report [9]. Also, we found that seizure is not gender related and previously proposed relation to younger ages is questionable. These observations are inconsistent with some previous reports $[25,26]$. Blood tramadol concentration was much higher in males maybe because males are more prone to drug dependency, abuse, and tolerance.

Although seizure happened in some cases with low blood tramadol levels, it did not occur in some cases with high blood tramadol concentrations. The reasons remained unclear, but drug dependency and tolerance may play a role in this regard. Applied methodology for translating pharmacokinetic data on toxicokinetic data could be imperfect. Additionally, the actual relationship between the dose and seizure could be weak. Tramadol which is used in Iran is an immediate release racemic product. There is no report in literature about any difference in kinetic between chronic users and nonchronic users. Tramadol is a racemic mixture of enantiomers of tramadol: $(+)$ and $(-)$ tramadol. Each of these enantiomers has different affinity for the mu and delta receptors and also their effects on the serotonin and norepinephrine reuptake. Therefore, depending upon their ratio, they affect seizure threshold differently. As a limitation of this study, the current analysis of GC method cannot distinguish between different isomers. On the other hand, since tramadol is metabolized in the liver and eliminated through the kidney, any renal or liver insufficiency may affect seizure threshold or incidence, but the present cases did not have those kinds of disorders. In addition, since metabolism of tramadol by the liver is prone to genetic polymorphisms, any co-ingested drug with potential to affect CYP enzymes will affect peak blood levels. This is also a concern that needs to be answered by further studies.

Tramadol is metabolized to its active metabolite, $o$ desmethyltramadol, plus multiple non-active metabolites.

$O$-Desmethyltramadol has a different affinity for the receptors and biogenic amine reuptake and may affect seizure threshold. Unfortunately, we cannot assay blood concentration level of $O$-desmethyltramadol in this study. It was another limitation of this study.

Formation of the $O$-desmethyltramadol metabolite is mediated mainly by cytochrom P450 2D6 (CYP2D6). CYP2D6 is the most highly polymorphic isoenzyme of the cytochrom P450-system.there is significant genetic polymorphism affecting the CYP enzymes in humans. This enzyme is deficit in about $10 \%$ of population. This enzyme genetic polymorphism may affect tramadol metabolism and peak blood levels. These pharmacodynamic issues (genetic polymorphism, different enantiomers, co-ingestion, different affinity to the receptors, and metabolism) could be the reason why we could not show a strong relation between tramadol blood level and occurrence of seizure in this study. Applying back-extrapolated dose to the time of exposure in poisoned patients is subjected to many confounders including the variable rate of absorption due to the ingestion of food, prior tolerance in addict cases, or potential interpersonal variability in regard to the kinetic study of tramadol. This model, however, was fixed according to kinetic data from a similar population. In addition, as concentration of tramadol was measured once, and time elapsed from exposure were different, this methodology is probably best estimates the true values.

In conclusion, tramadol-induced seizure is dose dependent. Gender and age of patients play no role in prevalence of seizures. Tramadol, similar to other opioids with unique structures such as dextropropoxyphene, which has shown to 
be much more fatal in overdose [31, 32] should be prescribed more cautiously particularly in opioid abusers and cases prone to intentional overdose and seizure.

Acknowledgments This study was supported by a grant from Tehran University of medical sciences. We thank Dr. Frederic J. Baud and Dr. Mostafa Jafarzadeh for their nice comments in editing the manuscript.

Declaration of Interest The authors report no declarations of interest. The authors alone are responsible for the content and writing of this paper.

\section{References}

1. Clarot F, Goullé JP, Vaz E, Proust B (2003) Fatal overdoses of tramadol: is benzodiazepine a risk factor of lethality. Forensic Sci Int 134(1):57-61

2. Musshoff F, Madea B (2001) Fatality due to ingestion of tramadol alone. Forensic Sci Int 116(2-3):197-199

3. Loughrey MB, Loughrey CM, Johnston S, O'Rourke D (2003) Fatal hepatic failure following accidental tramadol overdose. Forensic Sci Int 134(2-3):232-233

4. Michaud K, Augsburger M, Romain N, Giroud C, Mangin P (1999) Fatal overdose of tramadol and alprazolam. Forensic Sci Int 105(3):185-189

5. Shadnia S, Soltaninejad K, Heydari K, Sasanian G, Abdollahi M (2008) Tramadol intoxication: a review of 114 cases. Hum Exp Toxicol 27(3):201-205

6. Afshari R, Majdzadeh SR, Balali-Mood M (2004) Pattern of acute poisoning in Mashhad, Iran 1993-2000. J Toxicol Clin Toxicol 42 (7):965-975

7. Koushesh HR, Afshari R, Afshari R (2009) A new illicit opioid dependence outbreak, evidence for a combination of opioids and steroids. Drug Chem Toxicol 32(2):114-119

8. Afshari R, Tashakori A, Shakiba AH (2008) Tramadol overdose induced CPK rise, haemodynamic and electocardiographic changes and seizure. Clin Toxicol 46:369

9. Talaie H, Panahandeh R, Fayaznouri M, Asadi Z, Abdollahi M (2009) Dose-independent occurrence of seizure with tramadol. J Med Toxicol 5(2):63-67

10. Talaie H, Panahandeh R, Fayaznouri MR, Asadi Z, Abdollahi M (2008) Seizure associated with tramadol use/abuse. J Clin Toxicol $46: 628$

11. Kroenke K, Krebs EE, Bair MJ (2009) Pharmacotherapy of chronic pain: a synthesis of recommendations from systematic reviews. Gen Hosp Psychiatry 31(3):206-219

12. Hara K, Minami K, Sata T (2005) The effects of tramadol and its metabolite on glycine, gamma-aminobutyric acidA, and $\mathrm{N}$-methylD-aspartate receptors expressed in Xenopus oocytes. Anesth Analg 100(5):1400-1405

13. Rehni AK, Singh I, Kumar M (2008) Tramadol-induced seizurogenic effect: a possible role of opioid-dependent gammaaminobutyric acid inhibitory pathway. Basic Clin Pharmacol Toxicol 103(3):262-266

14. Sun C, Mtchedlishvili Z, Erisir A, Kapur J (2007) Diminished neurosteroid sensitivity of synaptic inhibition and altered location of the alpha4 subunit of GABA(A) receptors in an animal model of epilepsy. J Neurosci 27(46):12641-12650

15. Miura M, Saino-Saito S, Masuda M, Kobayashi K, Aosaki T (2007) Compartment-specific modulation of GABAergic synaptic transmission by mu-opioid receptor in the mouse striatum with green fluorescent protein-expressing dopamine islands. J Neurosci 27(36):9721-9728

16. Frenk H (1983) Pro- and anticonvulsant actions of morphine and the endogenous opioids: involvement and interactions of multiple opiate and non-opiate systems. Brain Res 287(2):197210

17. Kahn LH, Alderfer RJ, Graham DJ (1997) Seizures reported with tramadol. JAMA 278(20):1661

18. Raffa RB, Stone DJ Jr (2008) Unexceptional seizure potential of tramadol or its enantiomers or metabolites in mice. J Pharmacol Exp Ther 325(2):500-506

19. Hennies HH, Friderichs E, Schneider J (1988) Receptor binding, analgesic and antitussive potency of tramadol and other selected opioids. Arzneimittelforschung 38(7):877-880

20. Mehrpour O, Abdollahi M (2011) Poison treatment centers in Iran. Hum Exp Toxicol (in press)

21. Ardakani YH, Rouini MR (2007) Pharmacokinetics of tramadol and its three main metabolites in healthy male and female volunteers. Biopharm Drug Dispos 28(9):527-534

22. Spiller HA, Gorman SE, Villalobos D, Benson BE, Ruskosky DR, Stancavage MM, Anderson DL (1997) Prospective multicenter evaluation of tramadol exposure. J Toxicol Clin Toxicol 35 (4):361-364

23. Gholami K, Shalviri G, Zarbakhsh A, Daryabari N, Yousefian S (2007) New guideline for tramadol usage following adverse drug reactions reported to the Iranian Pharmacovigilance Center. Pharmacoepidemiol Drug Saf 16(2):229-237

24. Epstein DH, Preston KL, Jasinski DR (2006) Abuse liability, behavioral pharmacology, and physical-dependence potential of opioids in humans and laboratory animals: lessons from tramadol. Biol Psychol 73(1):90-99

25. Jovanović-Cupić V, Martinović Z, Nesić N (2006) Seizures associated with intoxication and abuse of tramadol. J Toxicol Clin Toxicol 44(2):143-146

26. Gardner JS, Blough D, Drinkard CR, Shatin D, Anderson G, Graham D, Alderfer R (2000) Tramadol and seizures: a surveillance study in a managed care population. Pharmacotherapy 20(12):1423-1431

27. Marquardt KA, Alsop JA, Albertson TE (2005) Tramadol exposures reported to statewide poison control system. Ann Pharmacother 39(6):1039-1044

28. Mehrpour M (2005) Intravenous tramadol-induced seizure: two case reports. Iran J Pharmacol Ther 4(2):146-147

29. Tjäderborn M, Jönsson AK, Hägg S, Ahlner J (2007) Fatal unintentional intoxications with tramadol during 1995-2005. Forensic Sci Int 173(2-3):107-111

30. Galer K, Krzyzanowski M (2004) Death attributed to toxic interaction of tramadol and other drugs. Arch Med Sadowej Kryminol 54(1):73-78

31. Afshari R, Good AM, Maxwell SR, Bateman DN (2005) Coproxamol overdose is associated with a 10 -fold excess mortality compared with other paracetamol combination analgesics. $\mathrm{Br} \mathrm{J}$ Clin Pharmacol 60(4):444-447

32. Bateman DN, Afshari R (2003) Co-proxamol and suicide: licence needs to be changed. BMJ 327(7409):287 